\title{
Alex Gómez
}

\section{Un productor multicultural}

\author{
Por Graciela Rodríguez-Milhomens \\ Foto archivo Alex Gómez
}

\section{RESUMEN}

Es un apasionado de la documentación audiovisual. Cuando estudiaba comunicación, en la compleja Cali colombiana de los noventa, Alex Gómez comenzó a documentar diferentes realidades, diferentes culturas, diferentes estilos de vida. Los trabajos que realizó con indígenas y comunidades afrocolombianas, varios de los cuales siguen siendo emitidos en la televisión de su pais, lo marcaron profundamente. Luego de trabajar en producción televisiva, estudió una maestría en educación en España y desde hace algún tiempo vive en Estados Unidos, pais que le ha permitido continuar con su pasión: la comunicación intercultural. Desde su empresa, dirige documentales, con la misma tónica: comunicar la diversidad, comunicar para la diversidad, comunicar para la interculturalidad.

Palabras clave: comunicación intercultural, identidad cultural, educación, producción audiovisual, documentales.

Su entrada al mundo de la producción audiovisual se dio, como pasa con muchos profesionales, cuando aprovechó, con talento, un momento de "buena suerte". Alex Gómez estudiaba el primer año de Comunicación Social, y trabajaba como mesero en un bar. "Un amigo invitó a una productora de televisión local a tomarse una cerveza, y yo le estaba sirviendo, y él me presentó. Ella me preguntó: '¿Estás estudiando comunicación?' Y yo le dije que sí. Se quedó callada; pero tres horas después me preguntó: '¿No te gustaría trabajar como asistente de producción?'. Súper intimidado le dije: 'Estoy en mi primer año de la universidad, todavía no tengo los

\section{ABSTRACT}

He is passionate about audiovisual documentaries. Alex Gómez began to record different realities, cultures and lifestyles already when he was a media scholar in the complex city of Cali during the 1990 's. He has been deeply influenced by his works on indigenous Afro-Colombian communities, many of which are still being shown on TV in that country. After working for TV in production units he undertook a Master in Education in Spain and he has been living in the United States where he can still develop his passion: intercultural communication. From his company he directs documentaries with the same spirit: communicating diversity, communication for diversity and communication for an intercultural world.

Key words: intercultural communication, cultural identity, education, audiovisual production, documentaries.

conceptos necesarios'. Y me dijo: 'Lo que necesitás para trabajar conmigo no lo vas a aprender en la universidad'. Dos meses después estaba trabajando como su asistente; afortunadamente ella era una de las mejores productoras audiovisuales en esa región. Aprendí mucho y muy rápidamente pasé a trabajar en una productora de televisión consolidada, como asistente de otra persona que también tenía un nombre público muy fuerte". Algunos de sus profesores de la Universidad del Valle, una de las más importantes de Colombia en comunicación, criticaban que Alex trabajara al tiempo que estudiaba: "Algunos me decian que 


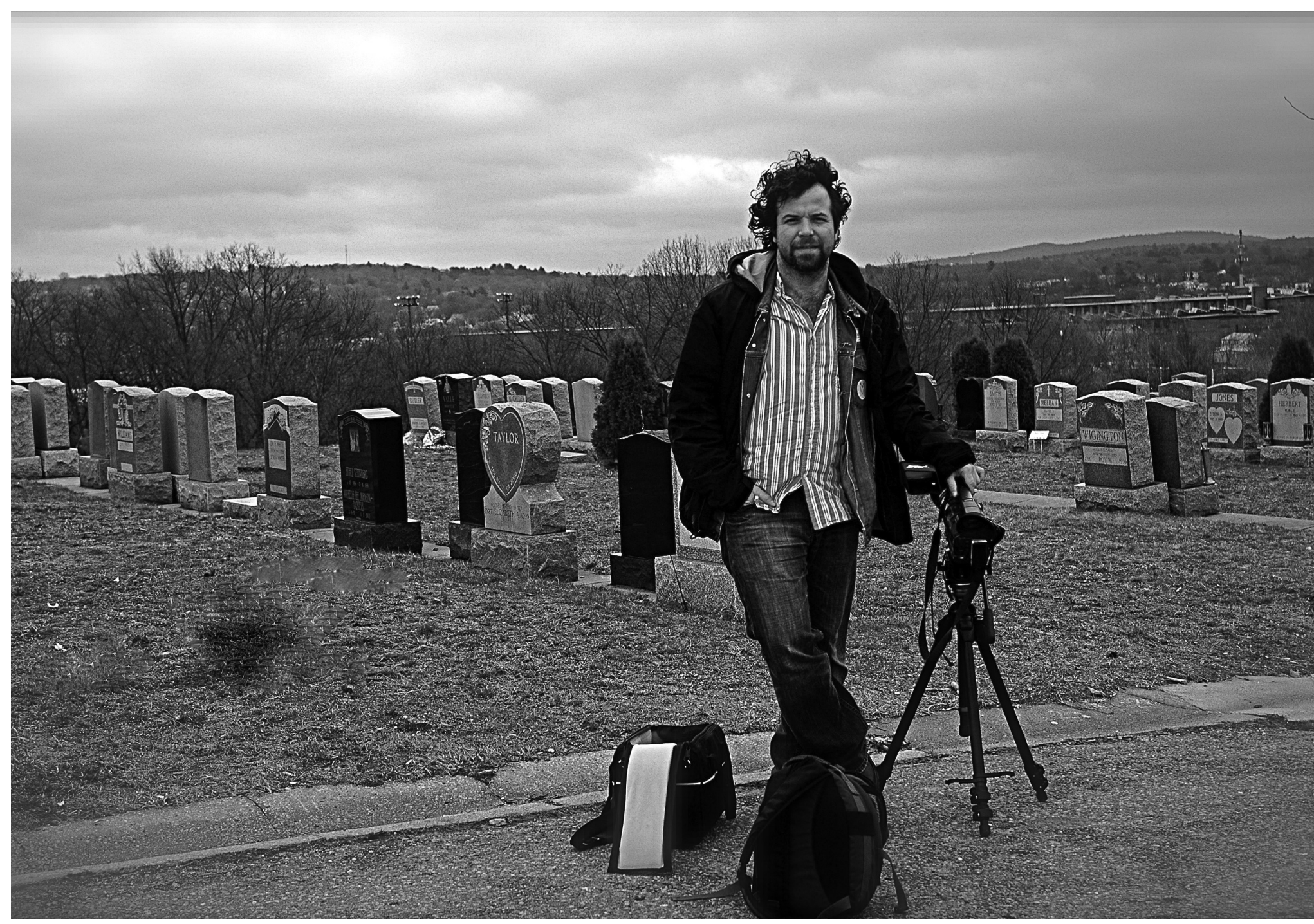

debería pasar más tiempo en la universidad, recibiendo la capacitación necesaria, pero la realidad es que uno aprende de diferentes maneras. En la universidad uno aprende conceptos, y en la vida real se enfrenta a situaciones reales en las que hay que tomar decisiones. Los dos conocimientos son válidos, creo yo. Y ambos me permitieron ser más independiente en el futuro".

Llegar a ser productor le costó algo de tiempo. Trabajó en asistencia de dirección en televisión, donde poco a poco empezó a dirigir algunos documentales. "Aquello era más complejo porque, además de manejar todos los presupuestos, tenía que manejar los contenidos, mirar cómo los iba a hacer... mucho trabajo. Pero todo esto fue vital para mi vida en los Estados Unidos porque hoy puedo diseñar y manejar tranquilamente presupuestos, y al mismo tiempo puedo trabajar en los contenidos con mis clientes".

A medida que ganaba autonomía en el trabajo, iba integrando temáticas sociales a su documentación. Empezó a trabajar con comunidades indígenas, afrocolombianas, en la zona del Pacífico. "Con este trabajo me sentía feliz conmigo mismo, estaba respondiendo a
Alex Gómez durante una producción de Intercultural Productions, en Dorchester, Massachusetts, 2007. 
Graciela RodríguezMilhomens::

(Montevideo, 1973) Magíster en

Comunicación (ITESO, México, 2008) y licenciada en Ciencias de la Comunicación Social (UCU, 1998). Es

consultora y directora en Tres Grupo Consultor. Es editora de dixit y profesora en la Maestría en Estudios

Organizacionales de la Universidad Católica del Uruguay. los intereses de la escuela donde fui, que eran intereses sociales, de reconocimiento de valores, de crear un concepto de identidad global basado en identidades más pequeñas". Las realidades en las que trabajó en aquellas épocas están plasmadas en documentales que aún hoy son emitidos en su país. Realidades complejas, sin lugar a dudas: "Algunas personas en estos lugares no se consideraban ni colombianas; obviamente los medios no se encargan de cubrir esa identidad que tienen, ni el gobierno se encarga de hacer presencia en esos lugares. Entonces era un trabajo muy interesante poder llegar allá y valorar a estos grupos, a estas comunidades, para después presentarle el documental al resto de Colombia. Es un trabajo que da satisfacción personal, hay que continuarlo con mucho tiempo para que realmente se cree esa identidad de que somos todos distintos, pero que todos somos parte".

Uno de sus documentales, La orilla, presenta un recorrido cultural por la historia afrocolombiana del Pacífico: "Empezamos en el sur del Pacífico colombiano y nos fuimos yendo hacia el norte, para ver cómo los ritmos africanos están presentes: ritmos en la música, en la vida, en las formas de pensar; ritmos presentes después de 370 años de haber llegado los africanos a territorio colombiano". En el año 1997, La orilla fue nominado como Mejor Documental en el Festival Internacional de Cine Premios India Catalina, y recibió una mención especial de la Comisión Nacional de Televisión (CNTV) en el Concurso Nacional de Televisión de Cartagena de Colombia. Otro de sus documentales, Carichipari, aborda un ritual indígena muy parecido al festejo de los 15 años de las chicas latinoamericanas: "Ellos tienen un ritual a los 13 años en el que presentan a las niñas en sociedad, un ritual donde emborrachan a sus niñas y algunas veces hasta terminan muertas; es bien complejo e intimidante. Con esas comunidades hay que hacer trabajos previos para poder acercarse, porque uno no puede llegar con una cámara a grabar". Trabajos de acercamiento que pueden llevar varios meses y requieren de muchos contactos, después de los cuales no necesariamente iniciaba el trabajo de grabación: "Muchas veces llegábamos a grabar y el gobernador de la comunidad no autorizaba la grabación, así que hacían un Cabildo y empezaban a discutir si podíamos grabar o no. Y ahí entraba yo para decirles que, en primer lugar, se iba a documentar su vida cotidiana y que ese trabajo les iba a servir para el futuro, porque es importante para una comunidad tener historia. Yo les garantizaba que el documento volvía a sus manos. Por otra parte, les decía que era una manera de que el resto de los colombianos los conociéramos, porque no teníamos ni idea de que estas culturas viven en medio de la selva. En esas discusiones a veces se nos iban dos meses antes de poder sacar una cámara”.

Otro documental importante y que tuvo un impacto importante en su país fue el que realizó por los 150 años de abolición de la esclavitud en Colombia. "Se trataba de un recorrido por varias comunidades afrocolombianas para ver en qué condiciones estaban hoy. Obviamente, no hay que entrar en una investigación muy detallada para darse cuenta de que estas comunidades muchas veces viven en peores condiciones que cuando eran esclavas. Cuando eran esclavos, el sistema económico garantizaba que el amo, el dueño de las tierras, les tenía que dar a ellos un lugar para vivir y les garantizaba la comida. Pero ahora, con la abolición, todo el mundo se desentendió y estas comunidades se quedaron sin las tierras que trabajaban y viviendo en peores condiciones que cuando las trabajaban siendo esclavos. Ese trabajo llamó mucho la atención y lo 

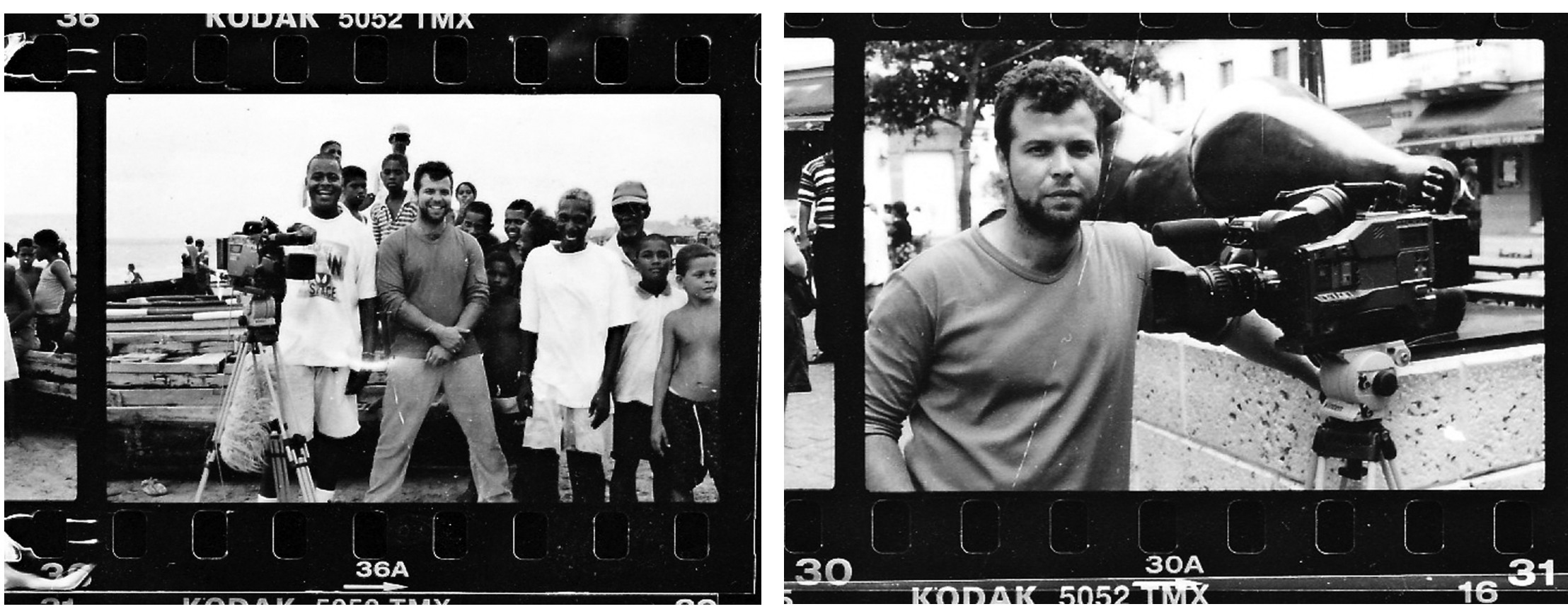

presentan todavía a nivel nacional de vez en cuando. Y también fue emitido por Televisión Educativa Iberoamericana".

\section{Boston y Nueva York}

La recuperación de las diferencias culturales como una riqueza fundamental encontró terreno fértil en Estados Unidos. Ciudades como Nueva York o Boston le permitieron encontrarse con otro tipo de diversidad, y fue, según dice, el mejor lugar para el nacimiento de su empresa productora, Intercultural Productions. "Te das cuenta de que ahí también hay una diversidad que viene de las diferentes nacionalidades, y que cada nacionalidad tiene, en sí misma, otras diversidades. Como comunicadores sociales necesitamos mirar procesos de comunicación... y ahí se dan muy bien porque hay muchos grupos: los latinos, los asiáticos, los anglosajones, todos tratando de relacionarse entre sí, sin tenerlo muy claro. Igual se mantienen grupitos que yo llamo 'burbujas'. Aquí, como en Colombia, busco realizar productos de comunicación que sirvan para crear diálogos entre estas comunidades".

Con esta intención inició con su productora, aunque al principio con un nombre que no funcionó del todo bien: "Mi intención era orientarme a organizaciones que trabajaran con comunidades en programas sociales, en el mejoramiento de la calidad de vida, en la disminución de la discriminación racial y cultural. Así que le puse a mi empresa Mystic Mirada, una mirada mística, pero nadie entendió qué era eso, aunque yo sí entendía mi mirada mística a todo ese proceso. Así que mi trabajo como comunicador empezó fallando al ponerle el nombre a mi empresa", señala, entre risas. Pero el asunto se resolvió un año y medio después, cuando Alex Gómez y su equipo se sentaron a pensar: "Este es un trabajo de comunicación: tenemos que ser claros con lo que hacemos"; hicieron una lluvia de ideas y surgió Intercultural Productions, "un nombre que abre las puertas por sí mismo porque no hay necesidad de que expliques nada". Fue a partir de este cambio que la productora comenzó a trabajar con fuerza: "Nos llamaron de la organización Latin American Health Institute, para realizar un video de sus programas, y desde ahí fue una bola de nieve: después vino el Boston Public Health Commission, para videos de educación que usan para enseñar a las comunidades, como por ejemplo el manejo de plagas para las casas que son asignadas por el gobierno de Boston".

En esta búsqueda de los diálogos interculturales, Alex Gómez encontró en la educación para la salud un área
Alex Gómez durante la producción de un documental en el Caribe Colombiano, 1999 
fundamental: "La salud y el mejoramiento de la calidad de vida es un punto de encuentro entre todas estas comunidades, porque todas tienen estas mismas necesidades: convivencia, en primer lugar; entendimiento cultural, porque obviamente todo el mundo viene de realidades distintas, entonces hay que buscar puntos de encuentro; y mejoramiento de la calidad de vida. Por ejemplo, el entendimiento de cómo funciona el sistema de salud de los Estados Unidos [un sistema complejo, por cierto] para que las personas puedan acceder a los beneficios. En fin, todas esas cosas están en lo que es la interculturalidad. Es como enseñar de nuevo a las personas, hacer que ellas entiendan, y no solamente a las personas, sino también a las instituciones. Es un trabajo de doble vía”.

\section{Completar la comunicación}

Hace un año y medio, una organización llamó a Intercultural Productions para plantearle un proyecto desafiante. En un barrio de Boston los jóvenes tenian altos indices de enfermedades de transmisión sexual, por lo que se empezó a trabajar para solicitar educación sexual en las escuelas. Relata Alex Gómez: "Me llamaron para que les dijera cómo podría ayudarles, desde el punto de vista comunicativo, para crear un documento que llamara suficientemente la atención de los medios y para que los profesores y padres de familia entraran en diálogo, con el objetivo de implementar clases de educación sexual en los salones de Boston. Todos los contenidos venían de los jóvenes. Después de varias semanas de trabajo, resolvimos que íbamos a hacer un video donde se presentara de manera muy tranquila y entretenida, una realidad que es mucho más seria que la manera en que está presentado el video". Fue así que realizaron el video con un formato de noticiero televisivo.

Quince minutos de testimonios, entrevistas a expertos y comerciales, en formato de informativo, sobre el mismo tema. "Este documento empezó a llamar la atención, no solamente a gente de aquí, sino de otros países... Lo pusieron en YouTube, empezaron todos los medios a fijarse, se involucraron políticos de relieve de Massachusetts, quienes empezaron a tomar estos trabajos de los estudiantes en nombre propio. Y fueron los políticos quienes comenzaron a pedir más clases de educación sexual para los jóvenes". El momento más interesante para ver el impacto del trabajo audiovisual, dice Alex Gómez, fue el momento en que la comunidad se reunió a ver el documental: "Vinieron 300 personas, entre políticos, profesores de las escuelas, estudiantes y padres de familia. Estaban ahí, claramente, los sectores que queríamos juntar, y ahí se dieron los temas de comunicación más importantes. Se armó una discusión muy interesante, hubo felicitaciones a los jóvenes, la gente se levantó a aplaudir. Y la discusión continuó de tal forma que la cadena CNN lo incluyó en su programación." Fue el momento del cierre del círculo de la comunicación: desde que se reunieron a pensar hasta que la CNN lo validó en su programación.

“El gobierno de los Estados Unidos está muy consciente de esa necesidad de la interculturalidad en todos los niveles. Si bien siempre ha sido un poco así, ahora es una política contundente”, señala Alex Gómez. Se trata de un asunto que involucra a la comunicación y a muchas otras disciplinas: "Se necesitan doctores que entiendan la multiculturalidad, se necesitan trabajadores sociales capaces de pasar de un concepto social a otro, capaces de dialogar. Se necesitan otras profesiones que asuman estas realidades. Desde la comunicación es posible encontrar estos puntos de contacto entre los grupos, para generar la discusión y el diálogo”.

\section{Las presiones en el trabajo}

En la producción audiovisual, como en cualquier campo, hay posibilidades de presiones relacionadas con el poder, 
situaciones que enfrentan a los profesionales a dilemas éticos concretos. En el caso de Alex Gómez, el trabajo independiente le da "mucha tranquilidad: yo puedo escoger qué temas están asociados a los valores y a la filosofía de mi empresa. De esta manera yo digo si me interesa o no. Se acercan negocios que necesitan hacer comerciales y evalúo mucho si lo que ellos están promoviendo tienen que ver lo que nosotros estamos haciendo". Recuerda el caso de un supermercado que se acercó pidiendo un comercial: "Nos reunimos, les hablé de que nuestra perspectiva es la interculturalidad. Entonces me dijeron que querían que en el comercial hubiera inclusión cultural y también de personas que tuvieran problemas físicos. Y ahí sí encontramos un punto para sentirnos cómodos: estas personas decidieron que querían poner una persona invidente dentro del comercial y a mí me pareció súper interesante. Así que dije: 'Bueno, del resto me encargo yo'. Quedaron muy contentos con su comercial que incluía gente de diferentes aspectos físicos y al señor invidente, porque querían dar el mensaje de que cualquier persona podía ir a ese supermercado. Eso me hizo sentir muy bien, desde el punto de vista ético. Así es como entiendo la labor social, aunque sea para un supermercado, porque al final todo el mundo tiene que ir a comprar a un supermercado".

Sin embargo, algunas veces fue más difícil mantenerse independiente, como en el caso de los documentales realizados en la montaña colombiana: "Una vez estábamos grabando en la montaña, cuando recibimos un mensaje de un grupo guerrillero que nos decía que teníamos que ir a grabar a un señor que estaba manejando mal sus tierras; mandaron a una persona con un fusil para decirnos esto. Obviamente, nosotros habíamos recibido algún tipo de entrenamiento para esos encuentros, así que le dijimos: 'Sí, nosotros terminamos esta información, vamos para allá y ahí gra- bamos lo que tengamos que grabar', porque no puedes ir en contra... Y me acuerdo que lo que hicimos ese día fue subirnos al carro y seguir derechito por donde habíamos llegado, no paramos para nada, íbamos súper miedosos. No pasó nada. No podemos negar que el miedo siempre existe, particularmente en esas zonas de conflicto, pero decidimos no ir para allá”.

Hay otro tipo de temor, menos extremo que el de la montaña, en el ejercicio profesional. Como es el caso de "potencialmente, ser capaces de equivocarse", dice Alex Gómez, "un temor que siempre tengo cuando asumo un proyecto. No sé si esto es general para todo el mundo, pero cada vez que yo recibo un proyecto, siento que están poniendo mucho en mis manos... y siempre me pregunto si mi intuición y mi conocimiento van a ser lo que el proyecto necesita. Pero, obviamente, yo sigo adelante. El temor siempre está y creo que es un temor necesario para aceptar la responsabilidad. Uno no puede estar tan seguro, porque estarías muy convencido de las cosas, y eso no es bueno. Obviamente que mis clientes no se dan cuenta de mi temor", asegura sonriendo. Un temor incómodo, sin dudas, pero también fundamental para estudiar, experimentar, aprender y aportar novedad.

Alex Gómez sigue trabajando cada día más. Su empresa, que inició en Boston, realizó varios proyectos en Nueva York y comienza a iniciar proyectos en Washington. Intercultural Productions representa un sueño que se materializó: "Yo necesitaba tener un proyecto con el que identificarme de por vida. Anduve en esa búsqueda desesperada durante años. Y, ciertamente, creo que Intercultural Productions es el comienzo de grandes cosas".

Un emprendedor siempre está buscando nuevas ideas para materializar. Sudamérica es, para él, un espacio para desarrollar. Ya llegará ese momento. :-: 